\title{
Microvascular blood flow in scalds in children and its relation to duration of wound healing: $A$ study using laser speckle contrast imaging
}

\author{
Robin Mirdell, Fredrik Iredahl, Folke Sjöberg, Simon Farnebo and Erik Tesselaar
}

\section{Linköping University Post Print}

\section{Tweet}

N.B.: When citing this work, cite the original article.

Original Publication:

Robin Mirdell, Fredrik Iredahl, Folke Sjöberg, Simon Farnebo and Erik Tesselaar, Microvascular blood flow in scalds in children and its relation to duration of wound healing: A study using laser speckle contrast imaging, 2016, Burns, (42), 3, 648-654.

http://dx.doi.org/10.1016/j.burns.2015.12.005

Copyright: Elsevier

\section{http://www.elsevier.com/}

Postprint available at: Linköping University Electronic Press

http://urn.kb.se/resolve?urn=urn:nbn:se:liu:diva-129497

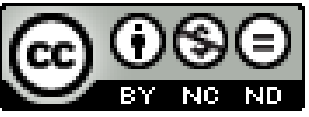




\section{Microvascular blood flow in scalds in children and its relation to duration of wound healing: a study using laser speckle contrast imaging}

Robin Mirdell $^{1}$, Fredrik Iredahl ${ }^{1}$, Folke Sjöberg ${ }^{1,2}$, Simon Farnebo ${ }^{1,2}$, and Erik Tesselaar ${ }^{1,3}$

1. Department of Clinical and Experimental Medicine, Linköping University, Linköping, Sweden.

2. Department of Hand and Plastic Surgery and Burns, Department of Clinical and Experimental Medicine, Linköping University, Linköping, Sweden.

3. Department of Radiation Physics and Department of Medical and Health Sciences, Linköping University, Linköping, Sweden

\section{Corresponding author:}

Erik Tesselaar

Department of Radiation Physics

Department of Clinical and Experimental Medicine

Linköping University

58185 Linköping, Sweden

tel. +46101038059

e-mail erik.tesselaar@liu.se

Running title: Blood flow changes in scalds in children 


\section{Abstract}

Background: Microvascular perfusion changes in scalds in children during the first weeks after injury is related to the outcome of healing, and measurements of perfusion, based on laser Doppler imaging, have been used successfully to predict the need for excision and grafting. However, the day-to-day changes in perfusion during the first weeks after injury have not to our knowledge been studied in detail. The aim of this study, based on a conservative treatment model where excision and grafting decisions were delayed to day 14 after injury, was to measure changes in perfusion in scalds using laser speckle contrast imaging (LSCI) during the first three weeks after injury.

Methods: We measured perfusion with LSCI in 34 patients at regular intervals between 6 hours after injury until complete reepithelialization or surgery. Duration of healing was defined as the time to complete reepithelialization.

Results: Less perfusion, between 6 and 96 hours after injury, was associated with longer duration of healing with the strongest association occurring between 72 and 96 hours. Burns that healed within 14 days had relatively high initial perfusion, followed by a peak and subsequent slow decrease. Both the maximum perfusion and the time-to-peak were dependent on the severity of the burn. Burns that needed excision and grafting had less initial perfusion and a gradual reduction over time.

Conclusion: The perfusion in scalds in children shows characteristic patterns during the first weeks after injury depending on the duration of wound healing, the greatest difference between wounds of different severity being on the $4^{\text {th }}$ day. Perfusion should therefore preferably be measured on the fourth day if it is to be used in the assessment of burn depth. 
Keywords: Scalds, burns, perfusion, laser speckle contrast imaging

Abbreviations: LDI=laser Doppler imaging, LSCI=laser speckle contrast imaging, PU=perfusion units and ROI=region of interest 


\section{Introduction}

The most common way to assess the depth of a burn is by looking at the wound and making a subjective assessment of capillary refill. Even though this method is immediate, easy and cost-effective [1], inaccurate prognoses are made in about a quarter of cases [2]. There is therefore a need for more reliable techniques for early prediction of the depth of a burn. The perfusion of blood in a superficial scald is typically higher than the perfusion of the surrounding uninjured skin, while a deeper scald often has a degree of perfusion similar to, or less than that of uninjured skin $[3,4]$. The perfusion of a scald has therefore been used as a diagnostic marker during assessment of the depth, which is of particular interest in intermediate burns as they are difficult for even experienced surgeons to diagnose correctly at initial presentation [5].

Several ways have been developed during recent years to measure perfusion objectively for the assessment of the depth of a burn and its healing potential, and these include near infrared spectroscopy, laser Doppler flowmetry (LDF), and laser Doppler imaging (LDI) [6]. Of those, only LDI is currently used clinically as a tool for the assessment of burns.

One of the major drawbacks of LDI is the time required to complete a scan of any larger area of skin, which is typically several minutes. As well as the practical disadvantage of the measurement taking a long time, LDI also tends to be affected by motion artefacts leading to inaccurate measurements, particularly in children [7]. Laser speckle contrast imaging (LSCI) is a recently developed technique that, like LDI, is able to measure perfusion in an area of skin, but within less time - typically a fraction of a second to a few seconds, depending on the image acquisition parameters, while at the same time maintaining high spatial resolution. These properties make LSCI a promising technique for the assessment of burns. 
We have previously shown [3] that perfusion measured with LSCI is higher in scalds that heal within 14 days than in wounds that require more than 14 days for reepithelialization, and this difference is already evident within the first 24 hours after injury.

In this study, in which we have included more scalds of various depths, we hypothesized that burns of differing severity and with different outcomes show evidence of specific perfusion dynamics during the first weeks after injury. Our aim was therefore to investigate how the perfusion in scalds in children (as measured by LSCI) changes during the first 15 days after the injury, and how this relates to the severity of the wound expressed as the time required for the wound to heal fully. 


\section{Patients and methods}

We invited all children with scalds who were admitted to the intensive burn care unit at Linköping University Hospital, or who were treated as outpatients, to participate in the study, and a total of 34 patients (14 of whom were female) were included. Their mean age was 2.7 (range 0.8 - 14.1) years. All patients initially received conservative treatment and a formal surgery decision was not made until 14 days after injury. Surgery was done when the treating physician predicted a wound area would require around 21 days after injury or more to heal. As all the children were under the age of consent, their parents or guardians gave permission for their participation in the study. The study was done in accordance with the Helsinki Declaration. The regional ethics committee approved the study on 22 February 2012, DNr 2012/31/31.

\section{Equipment}

We used a laser speckle contrast imager (Pericam PSI, Perimed AB, Järfälla, Sweden) to measure skin perfusion. The system uses a divergent laser beam with a wavelength of $785 \mathrm{~nm}$ to illuminate the skin, and creates a speckle pattern over the area that is illuminated. It uses two cameras, one that captures the speckle contrast image and the other that captures a conventional color image of the measured area. The principle of the technique of LSCI has been previously described in detail [3]. We set the image size to correspond to a $12 \mathrm{~cm} \mathrm{x} 12$ cm area of skin, kept the distance between the camera and the skin to between 18 and $27 \mathrm{~cm}$, and set the acquisition rate to 21 images/second. With each measurement, the system calculated the mean perfusion from 42 consecutive images, which resulted in a measurement time of 2 seconds for each scan. The spatial resolution of the perfusion image was 0.2 $\mathrm{mm} /$ pixel. The LSCI system was calibrated at regular intervals as recommended by the manufacturer. In 11 ROI in two patients, 5 subsequent scans were captured within a period of 1 minute to assess the reproducibility of the technique. 


\section{Measurement of perfusion}

All patients received sedatives during redressing of the wound in accordance with normal clinical practice. Usually this was a combination of midazolam and ketamine, with an addition of nitrous oxide occasionally. Propofol was however used in cases with more extensive scalds or when the patient reported side-effects from the ketamine. In a few cases, mostly older patients, satisfactory effect was achieved by nitrous oxide alone. We always measured the perfusion in the wound during the first visit directly after debridement and cleaning of the wound with sterile saline, which was between 6 and 72 hours after the injury. In most patients we then made additional measurements each time that the wound was dressed, typically every 2 to 4 days depending on the extent of the injury. Whenever possible we acquired images from the same areas of skin during subsequent visits so that we could follow changes in perfusion in the same wound over time. Some wounds were covered by biological dressings or xenografts (pigskin), and those were not always removed when the wound was dressed. As we have previously seen that the presence of these dressings has a large effect on the measurement of perfusion, we excluded those regions from the analysis. We also excluded injuries on the hands, feet, and cheeks, because we think that they could confound the data as a result of a relatively high and more variable baseline perfusion. In each image, we outlined regions of interest (ROI) using the LSCI software (PIMSoft 1.3, Perimed AB, Järfalla, Sweden). We often acquired several images from the same patient, and each image could have multiple ROI in case there were parts that clearly had different degrees of perfusion. For each region, the healing time was defined as the number of days needed for the region to reepithelialize completely. 
The time since injury was recorded in 6-hour intervals during the first day, and 24-hour intervals thereafter. A one-way analysis of variance was used to test whether there was a relation between duration of wound healing and the age of the patient. The differences in perfusion between wounds with different healing times was analyzed using unpaired, twotailed Student's $t$ tests with Welch's correction to compensate for differences in variance between groups. Data are expressed as mean (SD), and probabilities of less than 0.05 were accepted as significant. All statistical analyses were made with the aid of GraphPad Prism version 5.02 for Windows (GraphPad Software, San Diego California USA, www.graphpad.com). 


\section{Results}

We outlined 149 ROI that corresponded to areas of wounds in 34 patients (Table 1). We analyzed each ROI on 2-6 occasions, depending on the number of visits during which images had been acquired, which gave a total of 452 regions analyzed. There was no significant difference in the ages of patients with wounds of different severity $(p=0.59)$.

\begin{tabular}{|c|c|c|c|c|c|c|}
\hline & \multicolumn{2}{|c|}{ Number } & \multirow[t]{2}{*}{ Age (years) } & \multicolumn{3}{|c|}{ Perfusion (PU) } \\
\hline & patients & $R O I$ & & 6-12 hours & 18-24 hours & 72-96 hours \\
\hline \multicolumn{7}{|l|}{ Healed after } \\
\hline 3-4 days & 9 & 17 & $2.1(1.0-3.8)$ & $226 \pm 37 *^{\dagger}$ & $306 \pm 74 *^{\dagger}$ & $237 \pm 71{ }^{* \dagger}$ \\
\hline 5-7 days & 22 & 38 & $2.5(0.8-11.6)$ & $292 \pm 43 *^{\dagger}$ & $283 \pm 74 *^{\dagger}$ & $468 \pm 122 * \dagger$ \\
\hline 8-11 days & 21 & 49 & $2.4(0.8-14.1)$ & $217 \pm 35$ & $195 \pm 68 *^{\dagger}$ & $372 \pm 143 * \dagger$ \\
\hline 12-18 days & 12 & 21 & $3.9(1.3-14.1)$ & $148 \pm 10$ & $135 \pm 20$ & $171 \pm 150 *$ \\
\hline Surgery required & 7 & 24 & $2.5(0.9-4.4)$ & $145 \pm 13$ & $135 \pm 33$ & $108 \pm 33$ \\
\hline Total & 34 & 149 & $2.7(0.8-14.1)$ & & & \\
\hline
\end{tabular}

Table 1. The number of patients with scald burns included in the study, as well as the number of ROI, the median (range) age of the patients at the time of injury and the mean (SD) perfusion in the wound regions, for wounds of different severity. ${ }^{*}$ indicates a significant difference in perfusion compared with wounds that require surgery; $\dagger$ indicates a significant difference in perfusion compared with wounds that heal after 12-18 days.

Figure 1 shows the perfusion in burns categorized into groups of different severity (time until complete reepithelialization), measured within different time periods after injury. Generally, the perfusion was inversely related to duration of healing, with the exception of the least severe burns (healing time 3-4 days), in which the perfusion was relatively low 6-12 hours after the injury (226 $\pm 37 \mathrm{PU})$ and at $72-96$ hours after the injury (237 $\pm 71 \mathrm{PU})$. 


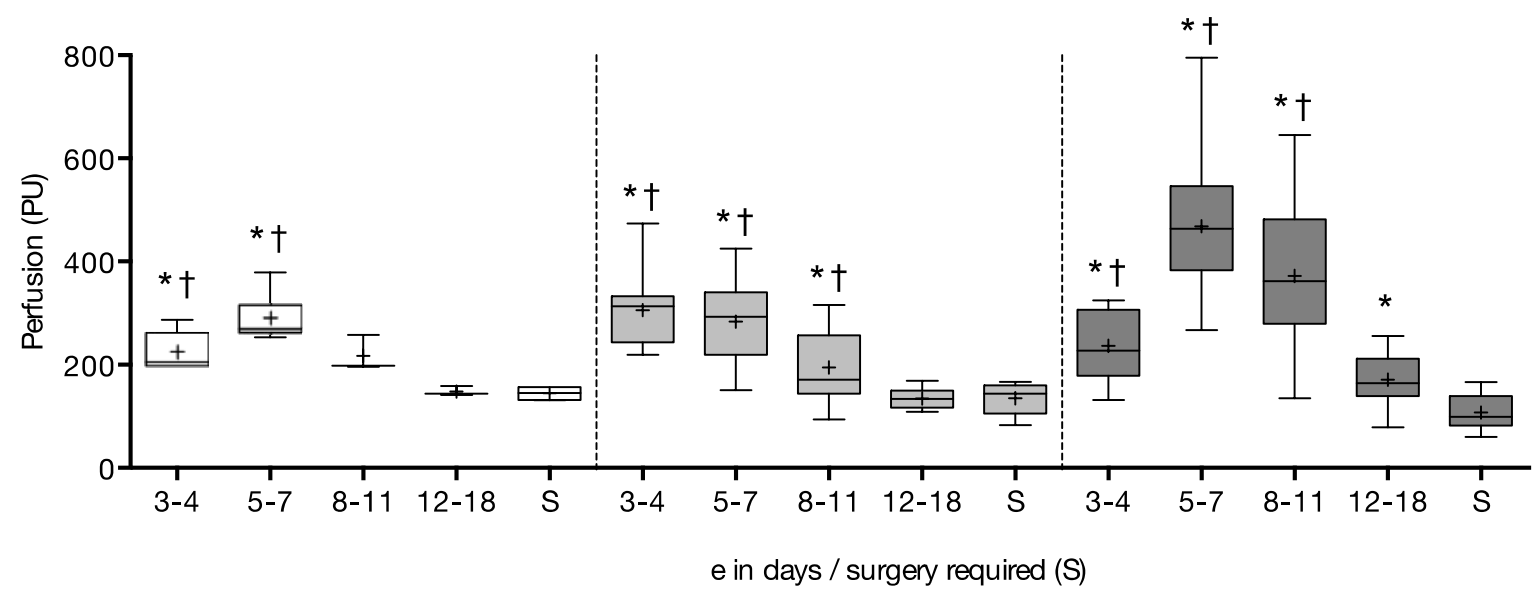

Figure 1. The effect of the severity of pediatric scald burns on wound perfusion, as measured using LSCI, at 6-12 hours, 18-24 hours and 72-96 hours post injury. * indicates a significant difference in perfusion compared with wounds that require surgery; $\uparrow$ indicates a significant difference in perfusion compared with wounds that heal after 12-18 days.

For clarity, the absolute perfusion values of wounds with different healing times measured within different time periods after the injury are listed in Table 1. Between 6 and 12 hours after injury, the burns that would heal within 7 days had significantly better perfusion than those that would heal after 12 days or would require surgery $(\mathrm{p}<0.007)$. Between 18 and 24 hours after injury, the burns that would heal within 11 days had significantly higher perfusion than those that would heal after 12 days or would require intervention $(\mathrm{p}<0.001)$. Between 24 and 72 hours after injury patients did not usually visit the burn clinic for redressing, which is why no analysis was made for that time period. Between 72 and 96 hours after injury the variability in the perfusion data was large in burns that would heal between 5 and 11 days, related to the considerable differences in perfusion dynamics between subgroups. However, burns that would heal spontaneously within 18 days had significantly better perfusion than those that would require excision and grafting $(\mathrm{p}<0.002)$. 

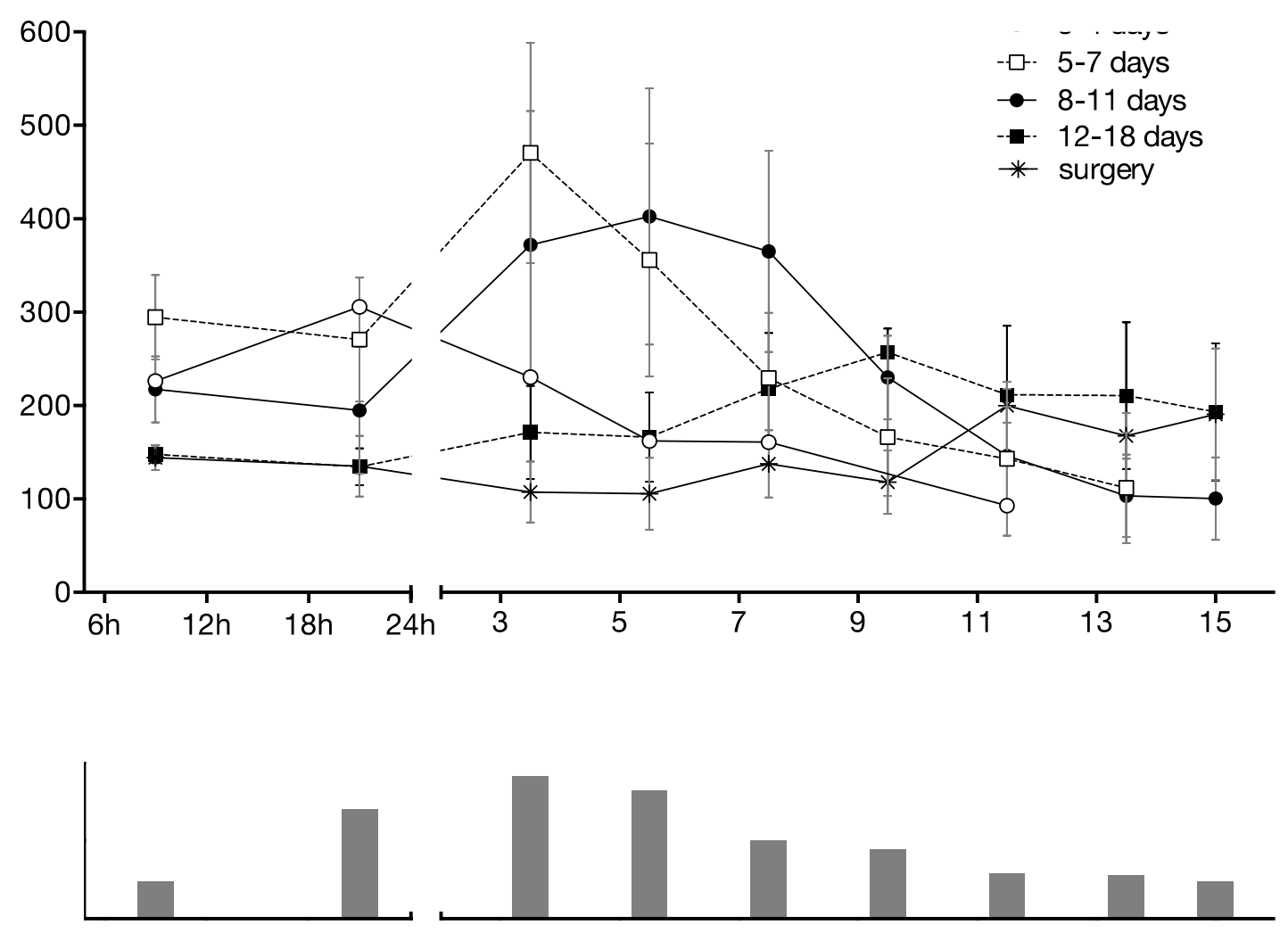

Figure 2. The mean (SD) perfusion in scalds of different severity in children, as measured using LSCI during the first 24 hours and subsequent 15 days after the injury. The lower panel shows the number of included wound regions (\# ROI) for each time interval.

Figure 2 shows the mean perfusion for different intervals of healing time between 6 hours and 15 days. Initially, wounds that would heal after 3-4 days showed among the best perfusion (226 \pm 37) PU), together with wounds that would heal after 5-7 days (292 \pm 43 PU). During subsequent days, wounds that would heal after 3-4 days showed a gradual reduction in perfusion approaching the minimum value by 11 days after the injury (93 $\pm 0 \mathrm{PU}$ ). Wounds that would heal after 5-7 days changed little over the first 24 hours, and then there was a remarkable increase in perfusion that reached its peak at 72-96 hours after the injury (471 \pm $116 \mathrm{PU})$. This was followed by a downward trend towards a perfusion of $112 \pm 59 \mathrm{PU}$ at 14 days. Wounds that would need 8-11 days to heal had a lower initial perfusion (217 \pm 34 PU) 
compared with wounds that healed between 5-7 days, and their perfusion decreased slightly until 18-24 hours. Their perfusion then increased rapidly to $403 \pm 137 \mathrm{PU}$ on the $5^{\text {th }}$ day, and thereafter decreased towards a level similar to that of the wounds with different healing times. Wounds that would heal between 12-18 days and those that would need surgery had the lowest initial perfusion values (146 $\pm 17 \mathrm{PU})$. Between 1 and 10 days after the injury it increased slightly, while the perfusion in those that would require surgery was unchanged, and lower during this period. After 10 days the perfusion in wounds that would heal after 12-18 days and in wounds that would need surgery converged towards $192 \pm 72 \mathrm{PU}$ at day 15 .

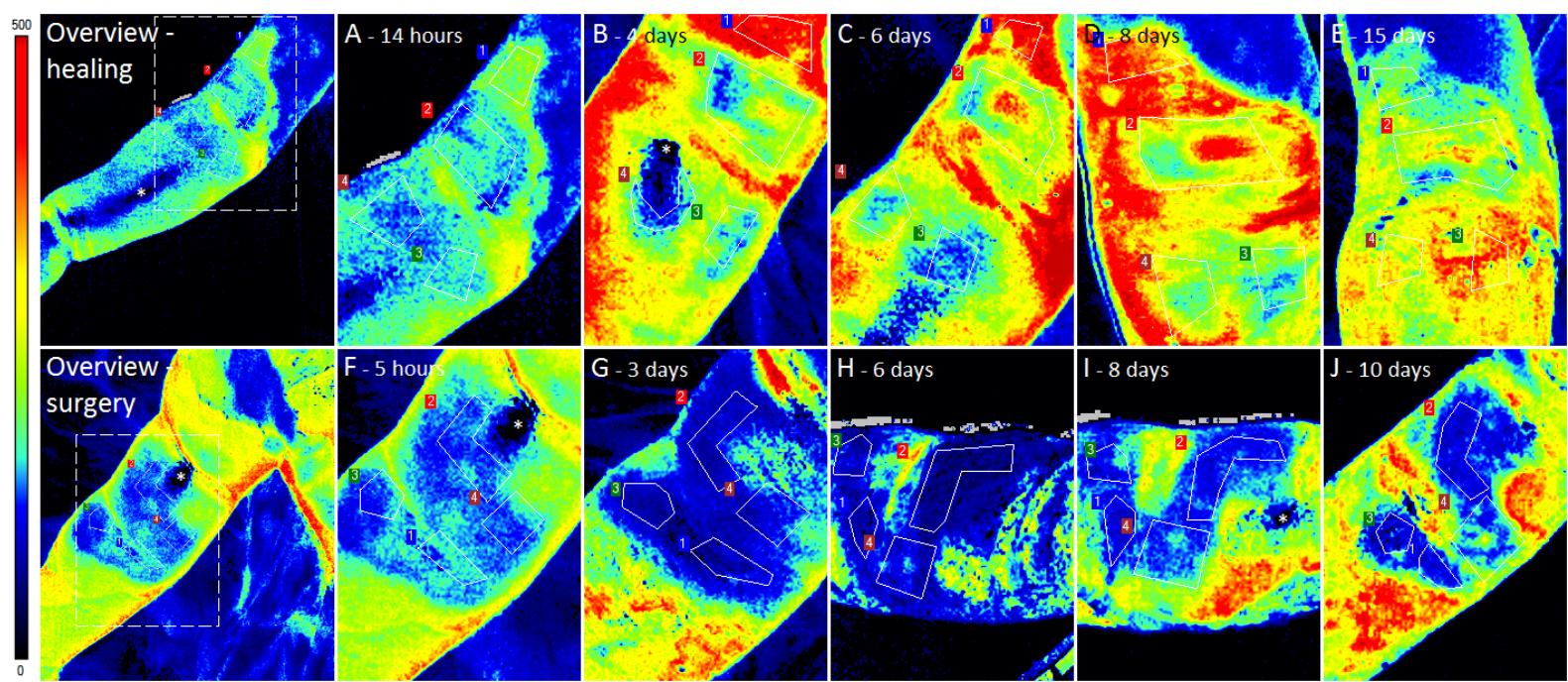

Figure 3. Examples of perfusion in scalds with various healing times in two different children. On the upper row, a wound on the right upper and lower arm of is shown which contains regions that healed between 9 and 17 days. Perfusion images A to E are acquired at 14 hours, 4 days, 6 days, 8 days and 15 days after the injury. On the lower row, a wound on the upper arm of another patient is shown, which contains different regions that did not heal after 14 days and subsequently required surgery. Perfusion images $\mathrm{F}$ to $\mathrm{J}$ are acquired at 5 hours, 3 days, 6 days, 8 days and 10 days after the injury. * indicates an area with erroneously low perfusion values due to specular reflections. The color bar on the left side indicates the perfusion scale (0-500 PU).

Figure 3 shows typical examples of wounds with various healing times in two patients. On the upper row is a wound on the right upper and lower arm that includes areas that healed between 9 and 17 days. Perfusion images A to E were acquired at 14 hours, 4 days, 6 days, 8 
days, and 15 days. On the lower row is a wound on the upper arm, which contains areas that did not heal after 14 days and were subsequently excised and grafted. Perfusion images $\mathrm{F}$ to $\mathrm{J}$ were acquired at 5 hours, 3 days, 6 days, 8 days, and 10 days.

In the 11 ROI for which subsequent images were acquired, the mean coefficient of variance of the perfusion value was $9.7 \%$. This value was obtained when including images with minor movement artefacts. 


\section{Discussion}

Our main finding was that perfusion in scalds in children, as measured by LSCI, shows characteristic dynamics during the first 15 days after injury. These depend on the duration of healing and the difference in perfusion between wounds that heal spontaneously within 15 days and wounds that need surgery, and is at its highest between 3 and 5 days after injury, which suggests that to predict outcome, perfusion should optimally be measured between 3 and 5 days after injury.

To the best of our knowledge, there has been only one previous study in which LSCI has been used to assess perfusion in burns, [3] and it showed that, perfusion measured with LSCI is greater in scalds that heal within 14 days than in wounds that require more than 14 days for complete reepithelialization. This difference was already significant on the first day after injury $(\mathrm{p}<0.001)$.

Several studies have investigated perfusion in burns using laser Doppler imaging (LDI). Hoeksema et al. measured the perfusion on different days after injury, [8] and found that the best interval to assess the depth using this method is between 3 and 5 days. Štětinský et al. found that outcome could not be predicted accurately by LDI until the $3^{\text {rd }}$ day [9].

The results of these studies agree with ours, even though they were of different burn types and not restricted to children. One advantage of studying scalds in children is their relatively uniform extent and distribution, as they typically cover parts of the neck, trunk, and arms. Another advantage is that when surgery is needed, it is usually done 14 days after injury allowing time for several measurements. This makes it possible to measure the perfusion in all patients and find out the degree of healing of the wound during the first two weeks after injury. 
We found remarkable differences in how perfusion changed over time in wounds of different severity. These patterns could be related to different degrees of vasoconstriction or vasodilation and subsequent microthrombosis, which have been reported within minutes of injury, and which potentially last up to 13 days depending on the severity of the wound [10].

The superficial wounds that would heal after 3-4 days were initially hyperemic, but perfusion gradually decreased from the first day after injury. This suggested that the provocation does not cause a prolonged extensive vasodilatory response, but only a limited degree of microthrombosis with little or no vascular damage.

Contrary to more superficial burns, the perfusion in those that were going to heal after 5-7 days increased considerably between the $1^{\text {st }}$ and $3^{\text {rd }}$ day after injury, the mean difference being as great as $234 \mathrm{PU}$ after 3-4 days. This may indicate two separate physiological processes in these otherwise almost equally severe groups of scalds, possibly related to differences in the temperature or the duration of the exposure to the hot liquid.

Vasodilation in wounds that would heal after 5-7 days and after 8-11 days was extensive, but both the time to maximum perfusion and the duration of the vasodilatory response increased with healing time, while the amplitude of the response decreased with healing time. The longer the time that was needed for these wounds to reach the maximum perfusion could be explained by moderate microthrombosis, which slows down the hyperemic response. The longer duration and lower amplitude of the hyperemic response in more severe wounds could be related to the greater degree of vascular damage and possibly the start of early angiogenesis [10].

We found no increase in perfusion before the $5^{\text {th }}$ and $10^{\text {th }}$ days after injury in either wounds that healed after 12-18 days or those that needed surgery. This is probably related to ongoing angiogenesis, which starts relatively early in deeper partial-thickness burns [11], 
where there is substantial occlusion of the microvascular bed as a result of microthrombi. In wounds that heal after 12-18 days the microthrombosis may be reversed earlier, because vascular damage is less extensive than in wounds that require excision and grafting.

The differences in how perfusion changes over time in wounds of differing severity may complicate the prediction of the need for intervention, particularly if a clinical decision is based on only a single measurement of perfusion. Some wounds that heal within 4 days have relatively low perfusion 2 days after injury. These, however, tend to be easy to classify correctly as superficial partial-thickness burns that can be treated conservatively. Wounds that require intervention initially have a perfusion similar to those that heal after 12-18 days, which means that if perfusion is measured too early, some wounds that will heal within 15 days may erroneously be thought to require surgery, and vice versa. In wounds in which perfusion is low within the first 24 hours we therefore recommend repeated measurements 3-4 days after injury.

In wounds that healed within 15 days there was a large variation in perfusion during the first week after injury. However, the variability in perfusion of wounds that required excision was low, which suggests that low perfusion, in particularly between 3 and 10 days after injury, may be a reliable indication that surgery is needed.

We noticed particular perfusion dynamics in the peripheral zone of burns that healed during the $2^{\text {nd }}$ week after injury or those that required excision and grafting. The perfusion in those regions generally varied like that in less severe wounds, but instead of a gradual decrease in perfusion to the degree measured in uninjured skin, these wounds maintained high perfusion even when the adjacent more severely burned areas were healing. This could be caused by cytokines and growth factors in the exudate that originated from the central wound, which caused a spillover signaling effect [12, 13]. Raised concentrations of heparinbinding EGF-like growth factor in the advancing epithelial margin could also cause an 
increase in perfusion [14]. Although not all wounds behaved in this way, we speculate that to some extent it occurs in all heterogeneous burns. We think that in such wounds it is important to look for the areas that have the lowest perfusion.

This study has a number of limitations. Most wounds were on the upper part of the torso, the arms, and the thighs. Perfusion in wounds on the hands, feet, and face differed from that in other areas, and we therefore chose to exclude them from the analysis. This means that care should be taken when applying the results reported here to other anatomical areas.

We did not investigate the effect of age on the changes in perfusion, but we found no obvious differences in the changes in perfusion with time between toddlers and older children.

When scalds have almost completely healed, patients tend to visit the burn clinic less frequently. We therefore had to estimate the exact healing time by looking at data before and after the wound had completely healed, and we think that the accurate healing time may differ by roughly 1 day. Patients' visits were also relatively uncommon on the second day after injury, which is why we did not analyze perfusion for that interval.

We did not take biopsy specimens or examine wounds histologically postoperatively, which means that we cannot rule out the possibility that they would have healed fully without scarring. However, because surgeries were done after 14 days, we were at least able to separate wounds that healed after 14 days from those that healed before. 14-21 days after injury is generally considered to be the cutoff time after which the risk for scarring increases substantially [15].

\section{Conclusions}

In conclusion, our results show that perfusion in scalds in children, as measured with LSCI, varies during the first 15 days after injury, and that the dynamics of perfusion depend on the 
severity of the wound. The difference in perfusion between wounds that heal within 15 days and wounds that needed excision and grafting is greatest between 3 and 5 days after injury. These findings should be borne in mind when LSCI is used for assessment of burns. 


\section{Acknowledgements}

We thank Ing-Marie Jarnhed, Matilda Karlsson, and Moustafa Elmasry for their help with the perfusion measurements. 


\section{References}

[1] Devgan L, Bhat S, Aylward S, Spence RJ. Modalities for the assessment of burn wound depth. J Burns Wounds 2006;5:e2. doi: 10.1016/j.burns.2008.01.009

[2] Heimbach D, Engrav L, Grube B, Marvin J. Burn depth: a review. World J Surg. 1992 JanFeb;16(1):10-5.

[3] Lindahl F, Tesselaar E, Sjöberg F. Assessing paediatric scald injuries using laser speckle contrast imaging. Burns 2013;39:662-6. doi:10.1016/j.burns.2012.09.018.

[4] Boykin JV, Eriksson E, Pittman RN. In vivo microcirculation of a scald burn and the progression of postburn dermal ischemia. Plast Reconstr Surg. 1980 Aug;66(2):191-8.

[5] Monstrey S, Hoeksema H, Verbelen J, Pirayesh A, Blondeel P. Assessment of burn depth and burn wound healing potential. Burns. 2008 Sep;34(6):761-9. doi: 10.1016/j.burns.2008.01.009.

[6] Kaiser M, Yafi A, Cinat M, Choi B, Durkin AJ. Noninvasive assessment of burn wound severity overusing optical technology: a review of current and future modalities. Burns. 2011 May;37(3):377-86. doi: 10.1016/j.burns.2010.11.012.

[7] Khatib M, Jabir S, Fitzgerald O'Connor E, Philp B. A systematic review of the evolution of laser Doppler techniques in burn depth assessment. Plast Surg Int. 2014;2014:621792. doi: $10.1155 / 2014 / 621792$.

[8] Hoeksema H, Van de Sijpe K, Tondu T, Hamdi M, Van Landuyt K, Blondeel P, Monstrey S. Accuracy of early burn depth assessment by laser Doppler imaging on different days post burn. Burns. 2009 Feb;35(1):36-45. doi: 10.1016/j.burns.2008.08.011.

[9] Štětinský J, Klosová H, Kolářová H, Šalounová D, Bryjová I, Hledík S. The time factor in the LDI (Laser Doppler Imaging) diagnosis of burns. Lasers Surg Med. 2015 Feb;47(2):196-202. doi: 10.1002/lsm.22291. 
[10] Yang Z. Chinese Burn Surgery. In: Springer Science+Business Media Dordrecht and People's Medical Publishing House 2015, 2.2 Thrombosis in Microvessels of Burned Skin p. 16-18. doi: 10.1007/978-94-017-8575-4.

[11] Pan SC, Wu LW, Chen CL, Shieh SJ, Chiu HY. Deep partial thickness burn blister fluid promotes neovascularization in the early stage of burn wound healing. Wound Repair Regen. 2010 MayJun;18(3):311-8. doi: 10.1111/j.1524-475X.2010.00586.x.

[12] Grad S, Ertel W, Keel M, Infanger M, Vonderschmitt DJ, Maly FE. Strongly enhanced serum levels of vascular endothelial growth factor (VEGF) after polytrauma and burn. Clin Chem Lab Med. 1998 Jun;36(6):379-83.

[13] Widgerow AD, King K, Tocco-Tussardi I, Banyard DA, Chiang R, Awad A, Afzel H, Bhatnager S, Melkumyan S, Wirth G, Evans GR. The burn wound exudate-an under-utilized resource. Burns. 2015 Feb;41(1):11-7. doi: 10.1016/j.burns.2014.06.002.

[14] McCarthy DW, Downing MT, Brigstock DR, Luquette MH, Brown KD, Abad MS, Besner GE. Production of heparin-binding epidermal growth factor-like growth factor (HB-EGF) at sites of thermal injury in pediatric patients. J Invest Dermatol. 1996 Jan;106(1):49-56. doi: 10.1111/1523-1747.ep12327214.

[15] Cubison TC, Pape SA, Parkhouse N. Evidence for the link between healing time and the development of hypertrophic scars (HTS) in paediatric burns due to scald injury. Burns. 2006 Dec;32(8):992-9. doi: 10.1016/j.burns.2006.02.007. 